\title{
The relation between cyclotron heating and energetic particles on open coronal field lines
}

\author{
R. Vainio ${ }^{\star}$ and T. Laitinen \\ Space Research Laboratory, Department of Physics, 20014 Turku University, Finland \\ Received 5 February 2001 / Accepted 16 March 2001

\begin{abstract}
Cyclotron resonance with high-frequency Alfvén waves has been proposed as an ion heating mechanism for producing high-speed winds and large ion temperatures in coronal holes. In the simplest version of the model (cyclotron sweep model), the waves propagate without interacting until they hit a resonance with the plasma ions at distance, where the ion cyclotron frequency becomes comparable to the wave frequency. We calculate the energetic test-particle mean free path $\lambda$ in solar corona heated by this mechanism. The primary result of our study is that energetic particles are efficiently coupled to the high-frequency MHD waves and may, thus, provide important feed back to wave-heating models. Detailed calculations are presented for a solar-wind stream of intermediate speed driven by the cyclotron sweep mechanism applying an extended quasi-linear theory for the mean free path. A local maximum of $\lambda$ is located near $r=2 R_{\odot}$, but $\lambda$ is very small at distances close to the Sun $\left(r<1.3 R_{\odot}\right)$ helping the acceleration of particles to high energies on open coronal field lines there. Another minimum for the mean free path is obtained near $r=10 R_{\odot}$ enabling ion acceleration beyond $10 \mathrm{MeV} / \mathrm{n}$ in streams of intermediate speeds by CME shock waves in the test-particle approximation. However, the presence of high-frequency Alfvén waves in amounts required for the cyclotron sweep heating has to be limited below $\sim 10 R \odot$ on field lines close to the ecliptic to avoid discrepancies with the inferred arrival times of solar energetic particles. Non-linear effects are discussed as a possible mechanism for removing the wave excess from the plasma.
\end{abstract}

Key words. Sun: corona - Sun: particle emission - solar wind

\section{Introduction}

The mean free path $\lambda$ of energetic particles due to their scattering off magnetic fluctuations in solar corona is an important parameter affecting the outcome of practically all models of energetic particle production on open coronal field lines. Whether one considers stochastic acceleration (Kocharov et al. 1999), shock acceleration (Vainio et al. 2000), or even acceleration in reconnecting magnetic fields (Kobak \& Ostrowski 2000), this parameter determines the typical energies attainable in the acceleration process, as well as the time scales for particle acceleration and the subsequent propagation. To be efficiently trapped by the turbulence to the acceleration process, the particle has to have a diffusion scale much smaller than the scale length of the acceleration region and the scale length of the ambient magnetic field, both of which are less or of the order of solar radius in corona. The relevant diffusion scale is the mean free path in the stochastic acceleration model and the diffusion length, $\kappa / u$, in case of shock acceleration,

Send offprint requests to: $\mathrm{R}$. Vainio,

e-mail: rami.vainio@utu.fi

* Supported financially by the Academy of Finland (project 46331). $\kappa=\frac{1}{3} \lambda v$ being the spatial diffusion coefficient, and $v$ and $u$ the energetic-particle speed and the (shock frame) upstream scattering-center speed, respectively.

In a weak turbulence theory, one can regard the fluctuations as being composed of low-frequency plasma waves. Observations in the interplanetary medium suggest that the most important wave mode present is the Alfvén wave propagating away from the Sun (e.g., Marsch 1991b). Using the standard quasi-linear theory (SQLT) to calculate energetic-particle mean free paths from wave-spectra, and extrapolating the observed interplanetary mean free paths in the interplanetary magnetic field, $B(r)$, back to the solar corona using the WKB Alfvén wave transport model yields (e.g., Ng \& Reames 1994)

$\lambda(r)=\lambda_{0}\left(\frac{B}{B_{0}}\right)^{q-1} \frac{V_{\mathrm{A} 0}}{V_{\mathrm{A}}}\left(\frac{V+V_{\mathrm{A}}}{V_{0}+V_{\mathrm{A} 0}}\right)^{q+1}$,

where $V$ and $V_{\mathrm{A}}$ are the solar wind and Alfvén speeds, respectively. For $\lambda_{0} \gtrsim 0.1 \mathrm{AU}$ and a turbulence spectral index $q=5 / 3$, both observed at $r_{0}=1 \mathrm{AU}$, this equation yields far too large values close to the Sun to be adequate for particle acceleration. Turbulent spectral evolution and dissipation of field fluctuations, however, can explain why the coronal mean free path can be substantially smaller 
than the value inferred from Eq. (1). On the other hand, if the small mean free path required for efficient particle acceleration extends far from the solar surface, it slows down the propagation of accelerated particles to $1 \mathrm{AU}$ too much to fit the observed small time lags between the solar flares/coronal mass ejections (CMEs) and particle events related to them.

The MHD-turbulence in the inner heliosphere was extensively studied by the two Helios missions (see Marsch 1991b for a review). It was found that indeed both dissipation and spectral evolution (that is, cascading) take place in the solar wind. The former is evidenced by the steepening of the fluctuation power spectrum near the proton cyclotron frequency. That the latter is operating in the solar wind is perhaps most clearly demonstrated by comparing the fluctuation power spectrum at distances from 0.3 to $1 \mathrm{AU}$ from the Sun. One can see a break in the spectrum between two power laws - spectral index $q=1$ for small frequencies and $q=5 / 3$ for larger ones - shifting towards lower frequencies with increasing distance from the Sun. This process has been confirmed to occur - although significantly slower - also in polar regions (Horbury et al. 1996), where waves propagate in a medium almost free of external forcing, like the velocity shear that disturbs their propagation in the ecliptic. Thus, interplanetary observations of the wave-power spectrum and energetic-particle mean free path do not restrict the turbulent conditions at high frequencies near the solar surface very much.

Dissipation of waves in corona and interplanetary medium is one of the mechanisms proposed for heating the corona and accelerating the solar wind. McKenzie et al. (1995) showed that the high speed wind and hot ions observed in the coronal holes could result from the damping of high-frequency Alfvén waves by cyclotron-resonant plasma ions. The origin of these waves divides the models of cyclotron heating into two classes (Cranmer 2000): one relying on (1) excitation of waves at the base of the corona (e.g., Tu \& Marsch 1997 and references therein), or (2) on continuous generation of high-frequency waves throughout the corona and solar wind (e.g., Hu et al. 1999 and references therein). Magnetic foot point motions and microflaring are possible processes launching such waves directly from the Sun. Models belonging to class (2) usually rely on a turbulent cascade where high-frequency waves are generated from lower-frequency waves in non-linear interactions. In this paper we will concentrate on class (1) for its simplicity, and models belonging to class (2) will be a subject of a future study.

Tu \& Marsch (1997) investigated the wave spectra that were needed to accelerate the wind to high speeds observed in coronal holes if the damping was due to the cyclotron sweep effect, where the waves would propagate non-interacting until they arrive at resonance with the ions; the resonance always occurs sooner or later due to the sweeping of the local cyclotron frequency towards lower values with increasing distance. The dissipation is assumed to occur instantaneously, when the wave hits the resonant dissipation frequency that decreases with the distance $r$ from the center of the Sun, i.e., when $f=f_{\mathrm{H}}(r)$. Tu \& Marsch (1997) take the dissipation frequency to be a fraction $\alpha_{\mathrm{f}}$ of the local proton gyrofrequency, $f_{\mathrm{H}}(r)=\alpha_{\mathrm{f}} \Omega_{\mathrm{p}}(r) / 2 \pi$. They consider initial wave spectra at the solar surface, $r=R_{\odot}$, that have a $f^{-1}$ behavior in four frequency bands, where the spectrum may have a different normalization. The initial spectrum at the lowest frequency range, from $\sim 10^{-4} \mathrm{~Hz}$ to $\sim 1 \mathrm{~Hz}$ is in agreement with low-frequency interplanetary observations. They show that a simple extrapolation of this spectrum up to the dissipation frequency would not be enough to produce wind speeds exceeding $700 \mathrm{~km} \mathrm{~s}^{-1}$. Thus, at $f>1 \mathrm{~Hz}$, their spectrum always has a higher normalization.

The purpose of this paper is to investigate the mean free path of energetic protons in a corona heated by the cyclotron sweep mechanism. It is not our intention to develop a new theory for the acceleration of energetic particles, but simply to demonstrate the effects the coronal wave-heating models may have on the transport and acceleration of energetic particles in solar corona. It is emphasized, that coronal heating, at least when it is based on high-frequency waves, should take into account the constraints of energetic-particle observations. As an example, we consider an intermediate-speed solar wind stream with a single $f^{-1}$ spectrum of outward-propagating Alfvén waves extending to a dissipation frequency near the local proton-cyclotron frequency. We employ the extended quasi-linear theory (EQLT) of Ng \& Reames (1995) to calculate the mean free path at distances below $0.3 \mathrm{AU}$ from the Sun. The result is discussed from the point of view of particle acceleration and transport, and reflected back to point out the constraints it sets on the coronal heating models.

\section{The model}

\subsection{The waves and the coronal medium}

We assume that the propagation of high-frequency Alfvénwaves from the solar surface up to the dissipation point is described by WKB theory. Thus, in a steady state for $f<f_{\mathrm{H}}(r)$, we get the power spectrum of the magnetic fluctuations, $P(f, r)$, from the conservation of the wave action,

$P(f, r) M(r)[M(r)+1]^{2}=P_{0}(f) M_{0}\left(M_{0}+1\right)^{2}$,

where $M(r)=V(r) / V_{\mathrm{A}}(r)$ is the Alfvénic Mach number of the radial flow, $V_{\mathrm{A}}=B_{r} /\left[4 \pi m_{\mathrm{p}} n\right]^{1 / 2}$ is the radial component of the Alfvén speed, $n(r)$ is the electron density, $B_{r}(r)$ is the radial component of the magnetic field, $m_{\mathrm{p}}$ is the proton mass, and $X_{0}(f) \equiv X\left(f, r_{0}\right)$ denotes the reference value of parameter $X$ at distance $r_{0}$. At the distance $r$, where the wave's (conserved) frequency hits the dissipation frequency the wave is absorbed by the plasma protons. We use the initial wave spectrum $P\left(f, R_{\odot}\right)=P_{\odot} / f$ with $P_{\odot}=10^{-3} \mathrm{G}^{2}$ in agreement with low-frequency interplanetary observations. 
Tu \& Marsch (1997) calculated the wave propagation self-consistently, i.e., using the wave heating function

$Q(r)=-\left(V+V_{\mathrm{A}}\right) \frac{P\left(f_{\mathrm{H}}, r\right)}{4 \pi} \frac{\mathrm{d} f_{\mathrm{H}}}{\mathrm{d} r}$

in proton internal energy equation and the wave-pressure gradient in the momentum equation as agents of coronal heating and solar wind acceleration, and then computing the steady-state solutions to the fluid equations. We simply pick up their solutions for the fluid behavior. We study their Model 1, which consists of a radial magnetic field

$B_{\mathrm{r}}=1.3\left(\frac{R_{\odot}}{r}\right)^{2} \mathrm{G}$,

and a density profile that we fit approximately by

$$
\begin{aligned}
n= & \sum_{j} n_{j}\left(\frac{R_{\odot}}{r}\right)^{j} \mathrm{~cm}^{-3}, \text { with } \\
& n_{16}=1.710^{8}, n_{8}=2.810^{7}, \\
& n_{3}=1.210^{6}, n_{2}=1.810^{5}, \text { and } \\
& n_{j}=0, \text { otherwise. }
\end{aligned}
$$

The equation of continuity can be written as

$\frac{n V}{B_{\mathrm{r}}}=\frac{n_{0} V_{0}}{B_{\mathrm{r} 0}}$

which determines $V(r)$ once its value is fixed at a reference point. From $\mathrm{Tu} \&$ Marsch (1997), we adopt $V_{0}=$ $550 \mathrm{~km} \mathrm{~s}^{-1}$ at $r_{0}=65 R_{\odot}$. Finally, we note that the proton thermal speed, $v_{\mathrm{p}} \equiv\left[2 k_{\mathrm{B}} T_{\mathrm{p}} / m_{\mathrm{p}}\right]^{1 / 2}$, for the proton temperature profile $T_{\mathrm{p}}(r)$ of $\mathrm{Tu} \&$ Marsch (1997) can be crudely fitted by

$v_{\mathrm{p}} \approx 90\left(\frac{r}{R_{\odot}}\right)^{2} \frac{1}{\left[1+\left(2 r / 3 R_{\odot}\right)^{4.35}\right]^{1 / 2}} \mathrm{~km} \mathrm{~s}^{-1}$.

The speeds $V, V_{\mathrm{A}}$, and $v_{\mathrm{p}}$ are plotted in Fig. 1 along with the rms amplitude (per logarithmic bandwidth) of the Alfvén waves, $\delta V=V_{\mathrm{A}}[f P(f, r)]^{1 / 2} / B_{\mathrm{r}}$.

\subsection{Dissipation frequency}

The cyclotron damping rate of Alfvén waves propagating parallel to the field lines is (e.g., Stix 1962)

$\gamma(f, r)=\Omega_{\mathrm{p}} \frac{\sqrt{\pi}}{2} \frac{f_{\mathrm{p}}}{\beta_{\mathrm{p}}^{1 / 2} f} \exp \left\{-\frac{f_{\mathrm{p}}^{2}}{\beta_{\mathrm{p}} f^{2}}\right\}$,

where $f_{\mathrm{p}}=\Omega_{\mathrm{p}}(M+1) / 2 \pi$ is the Doppler-shifted protoncyclotron frequency, $\beta_{\mathrm{p}}=8 \pi n k_{\mathrm{B}} T_{\mathrm{p}} / B^{2}$ is the proton plasma beta, $T_{\mathrm{p}}$ is the proton temperature, and $k_{\mathrm{B}}$ is Boltzmann's constant. Assuming that the spectrum terminates at that frequency, where the damping length, $\left(V+V_{\mathrm{A}}\right) / 2 \gamma$, equals the distance from the surface of the Sun, $r-R_{\odot}$, gives an equation

$\Omega_{\mathrm{p}} \sqrt{\pi} \frac{f_{\mathrm{p}}}{\beta_{\mathrm{p}}^{1 / 2} f_{\mathrm{H}}} \exp \left\{-\frac{f_{\mathrm{p}}^{2}}{\beta_{\mathrm{p}} f_{\mathrm{H}}^{2}}\right\}=\frac{V+V_{\mathrm{A}}}{r-R_{\odot}}$

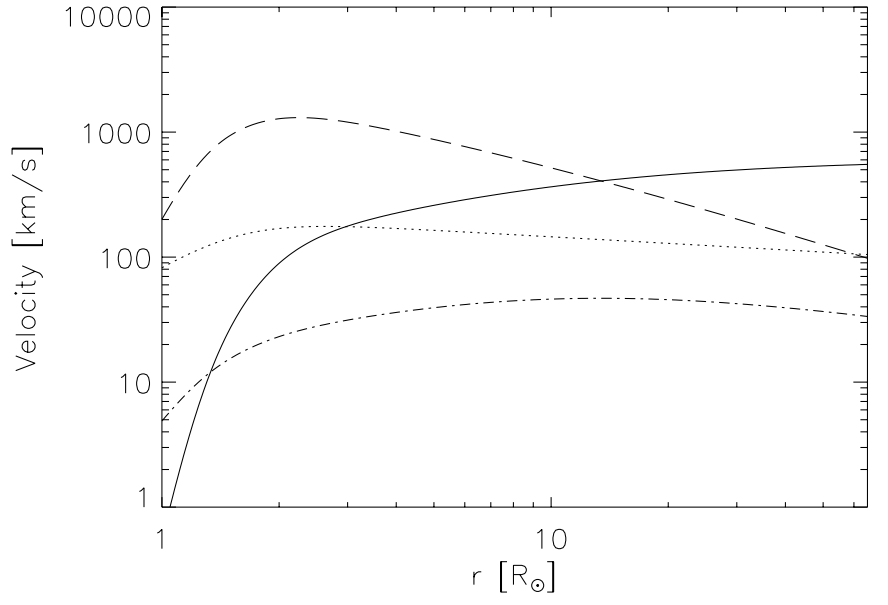

Fig. 1. Solar wind speed (solid curve), Alfvén speed (dashed curve), proton thermal speed (dotted curve), and rms amplitude of the Alfvén waves (dash-dotted curve) in a corona heated by high-frequency Alfvén waves. See text for details

that can be accurately solved for $\beta_{\mathrm{p}}^{1 / 2} f_{\mathrm{H}} / f_{\mathrm{p}}$ by iteration as

$\frac{f_{\mathrm{H}} \beta_{\mathrm{p}}^{1 / 2}}{f_{\mathrm{p}}} \approx\left[\ln \frac{Y}{\sqrt{\ln Y}}\right]^{-1 / 2}, Y(r)=\sqrt{\pi} \frac{\Omega_{\mathrm{p}}\left(r-R_{\odot}\right)}{V+V_{\mathrm{A}}}$.

One has to check that the termination frequency does not increase with distance, since no spectral replenishment is thought to occur. Further, we do not want to consider waves that are too much affected by dispersive effects, so we take

$f_{\mathrm{H}}(r) \leftarrow \min \left\{f_{\mathrm{H}}(r), 0.5 f_{\mathrm{p}}(r)\right\}$

The resulting spectral termination frequency with the above steps elucidated is plotted in Fig. 2.

The exact way of taking cyclotron damping into account is to multiply the right hand side of Eq. (2) by

$\exp \left\{-\int_{r_{0}}^{r} \mathrm{~d} r^{\prime} \frac{2 \gamma\left(f, r^{\prime}\right)}{V\left(r^{\prime}\right)+V_{\mathrm{A}}\left(r^{\prime}\right)}\right\}$.

We have numerically integrated the damping decrement and found the analytical approximation (Eq. (4)) for the cutoff frequency very reasonable. In addition, the Mach number is small at $r<10 R_{\odot}$ so that there $f_{\mathrm{H}} \approx \alpha_{\mathrm{f}} \Omega_{\mathrm{p}} / 2 \pi$ with $\alpha_{\mathrm{f}}=0.5$. Note that the value of $\alpha_{\mathrm{f}}$ does not affect the heating rate for a $1 / f$ wave spectrum, so adopting this value instead of $\alpha_{\mathrm{f}}=0.1$ used by Tu \& Marsch (1997) does not lead to inconsistencies, provided that we scale their spectral breakpoint frequencies by 5 , accordingly.

\subsection{Energetic-particle mean free path}

Energetic-particle mean free path $\lambda$ is related to the pitchangle diffusion coefficient $D_{\mu \mu}$ by (e.g., Schlickeiser 1989)

$\lambda=\frac{3 v}{8} \int_{-1}^{+1} \frac{\left(1-\mu^{2}\right)^{2}}{D_{\mu \mu}} \mathrm{d} \mu$. 


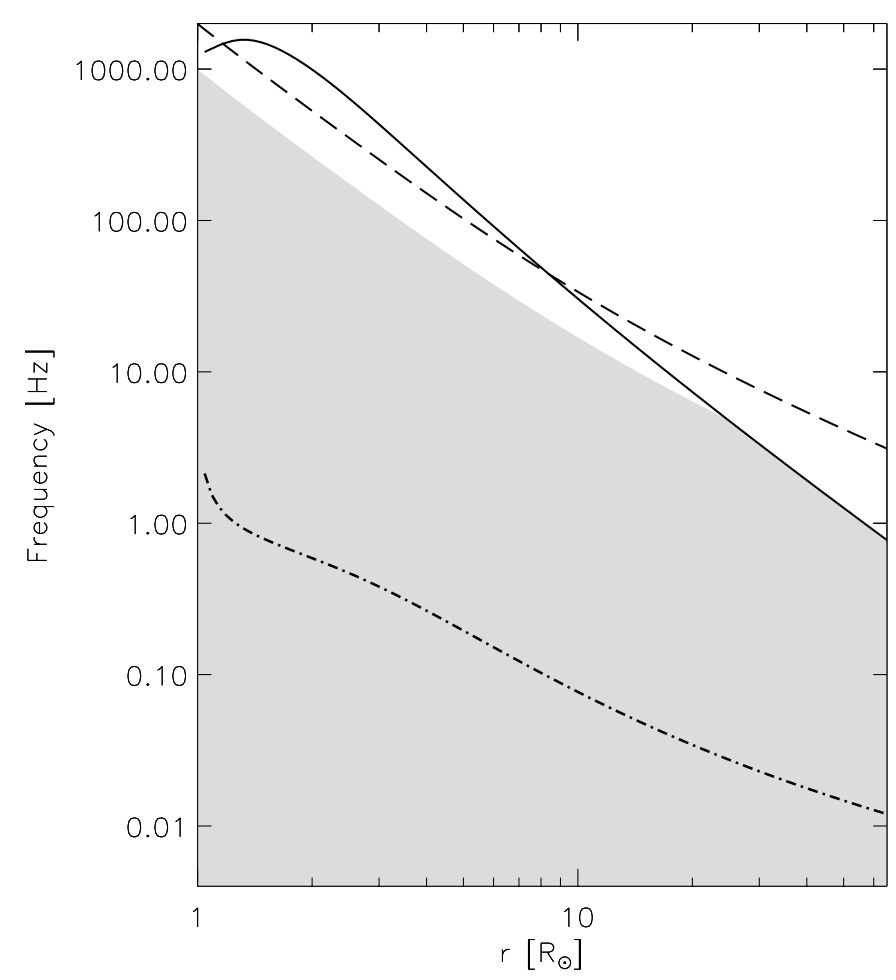

Fig. 2. Frequency range (shaded area) for Alfvén waves used in our model, extending from $f_{0}=10^{-4} \mathrm{~Hz}$ (not shown) up to the dissipation frequency $f_{\mathrm{H}}$. The curves show the iteration result (Eq. (4), solid curve), the Doppler shifted protoncyclotron frequency $f_{\mathrm{p}}$ (dashed curve), and the maximum frequency for phase-correlated Alfvén waves, $f_{\mathrm{c}}=k_{\mathrm{c}}\left(V+V_{\mathrm{A}}\right) / 2 \pi$ (dash-dotted curve), see Eq. (A.1)

The SQLT form of $D_{\mu \mu}$ due to a spectrum of parallelpropagating Alfvén-waves for super-Alfvénic $\left(v \gg V_{\mathrm{A}}\right)$ protons is given by

$D_{\mu \mu}^{\mathrm{SQLT}}=\frac{\pi}{2} \frac{\Omega^{2}}{B^{2}}\left(1-\mu^{2}\right) \frac{I(-\Omega / v \mu)}{|v \mu|}$,

where $\mu, v$, and $\Omega=e B /\left(\gamma m_{\mathrm{p}} c\right)$ are the pitch-angle cosine, speed, and gyro-frequency of the particle (all measured in the wave frame). Here, $I(k)$ is the wavenumber spectrum of the Alfvén waves, where positive (negative) values of the wavenumber $k$ denote left-handed (right-handed) helicity. It is evaluated in each point of velocity space at a single, resonant wavenumber $k=-\Omega / v \mu$.

For outward-propagating Alfvén waves, $2 \pi f=|k|(V+$ $\left.V_{\mathrm{A}}\right)$, and in case of vanishing net magnetic helicity, $I(-k)=I(k)$, we may write

$|k| I(k, r)=\frac{1}{2} f P(f, r)$,

which, for a spectrum of the form $P(f, r)=R(r) / f$, yields a particularly simple form

$D_{\mu \mu}^{\mathrm{SQLT}}=\frac{\pi}{4} \Omega\left(1-\mu^{2}\right) \frac{R(r)}{B^{2}}$.

This applies for $|\mu|>\mu_{\mathrm{c}} \equiv \Omega /\left(v k_{\mathrm{H}}\right)$, where $k_{\mathrm{H}}=$ $2 \pi f_{\mathrm{H}} /\left(V+V_{\mathrm{A}}\right)$ is the highest wavenumber in the spectrum.
Inside $|\mu|<\mu_{\mathrm{c}}$, SQLT has to rely on ion-cyclotron waves to scatter the particles. Assuming the cold-plasma dispersion relation for the waves, Vainio (2000) showed that there is no resonance gap provided that cyclotron waves propagating in both directions are present with a spectral index $q<6$; otherwise, SQLT would be an inadequate description of particle scattering across $\mu=0$. In the model of Tu \& Marsch (1997), neither of these constraints is fulfilled. Thus, there are no resonant waves to provide scattering across $\mu=0$, and particles must find other ways to cross it. Different non-resonant processes, like resonance broadening, mirroring and non-linear effects can provide scattering across $\mu=0$ for fast enough particles. To estimate the non-resonant corrections, several models have been presented in literature. Resonance broadening due to finite wave-particle interaction times caused either by thermal damping (Schlickeiser \& Achatz 1993) or by turbulent spectral evolution (Bieber et al. 1994) would be one option. In the present model, however, turbulent spectral evolution is neglected, and thermal damping does not produce enough resonance broadening: it would require waves with large damping rates to be present in the spectrum but in our model, waves with damping rates larger than $\left(V+V_{\mathrm{A}}\right) /\left(r-R_{\odot}\right)$ are absent.

In the extended quasi-linear theory of $\mathrm{Ng} \&$ Reames (1995), one obtains resonance broadening by including (non-resonant) medium-scale fluctuations, $\Delta \boldsymbol{B}$, of the magnetic field to the equation of motion of particles, and then ensemble averaging the resulting forces over the gyro phase of the particles, as well as the gyro phase and magnitude of these fluctuations. This turns out to be a very efficient process, since effectively, the pitch-angle diffusion coefficient of SQLT gets smoothed with a function of half width $\sim b_{\perp} \equiv \Delta B / B$. In addition, a broadened Čerenkov resonance appears. The EQLT mean free path is obtained by numerically integrating the pitch-angle diffusion coefficient calculated in Appendix A, i.e.,

$\lambda=\frac{3 r_{\mathrm{L}}}{2} \int_{0}^{1} \frac{1-\mu^{2}}{\sum_{n=0}^{2} \Phi_{n}(\mu, v)} \mathrm{d} \mu$,

with $\Phi_{n}(\mu, v)$ given by the Eqs. (A.2), (A.5), and (A.6) for $n=0,1$, and 2 , respectively. EQLT is adequate for the description of particle transport only for $\mu_{\mathrm{c}}<2 b_{\perp}$; otherwise processes not included in our simplified treatment have to be included to describe scattering near $\mu=0$. To estimate $b_{\perp}$, we use the part of the wave spectrum that extends from a minimum wavenumber corresponding to a distance of unperturbed motion along the field, $k_{\min }=2 \pi /(\lambda|\mu|)$, to maximum wavenumber at resonant scales. For simplicity, we take the SQLT values for these two parameters, i.e., $k_{\min }=\pi^{2} R(r) /\left(B^{2} r_{\mathrm{L}}|\mu|\right)$ and $k_{\max }=1 /\left(r_{\mathrm{L}}|\mu|\right)$. This is reasonable, as long as the resulting EQLT mean free path is not too different from the SQLT value. Thus,

$b_{\perp}^{2}=\frac{R(r)}{B^{2}} \ln \frac{B^{2}}{\pi^{2} R(r)}$.

In case the logarithm is smaller than 1 , we replace it by unity. 


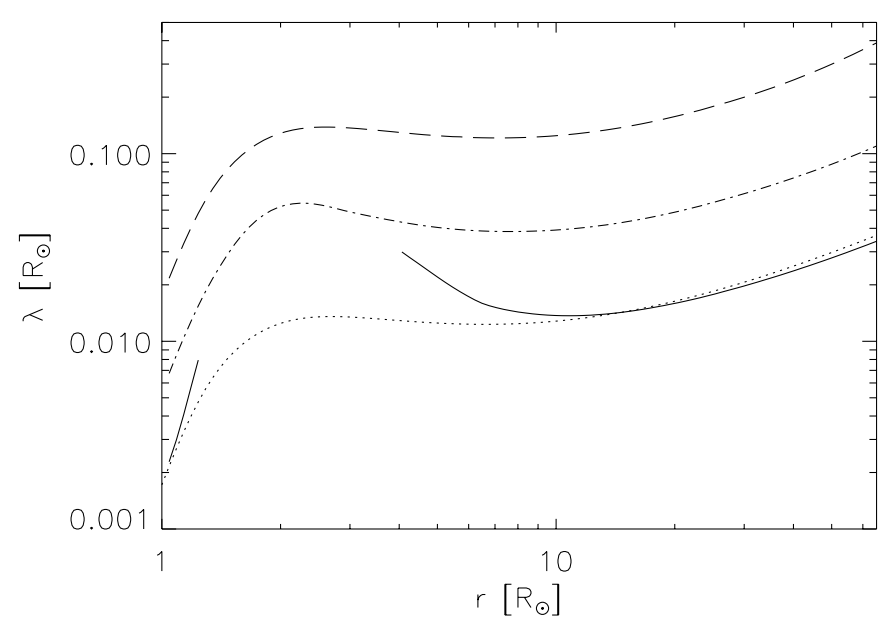

Fig. 3. The EQLT mean free path as a function of distance in solar corona for energies $E=1$ (solid curve), 10 (dash-dotted curve), and $100 \mathrm{MeV}$ (dashed curve). The resonant part of the $1-\mathrm{MeV}$ mean free path is also shown (dotted curve)

\section{Results and discussion}

In Fig. 3, we have plotted the mean free path of energetic protons in corona as a function of distance for three energies: $E=1,10$, and $100 \mathrm{MeV}$. For comparison, we have plotted also the SQLT mean free path for $1 \mathrm{MeV}$ energy, and we see that where it can be applied, the EQLT mean free path is, at most, a couple of times the SQLT value. The curves show a local maximum of the mean free path around $2 R_{\odot}$, where the Alfvén speed is large and the Mach number is still much less than unity (see Eq. (1)). Between 1.3 $R_{\odot}<r<4 R_{\odot}$, the amplitude of the Alfvén waves is too small to allow the closure of the resonance gap at $1 \mathrm{MeV}$ by EQLT, and in this region the mean free path may be considerably larger than the SQLT prediction.

The mean free path as a function of kinetic energy is plotted in Fig. 4 at three distances, $r=2$, 5, and $10 R_{\odot}$. For the largest distance, we have also included the SQLT value as a separate curve. The mean free path at $2 R_{\odot}$ shows a flattened behavior at $E<20 \mathrm{MeV}$ and goes abruptly to infinity at $E \approx 4 \mathrm{MeV}$ indicating the invalidity of EQLT treatment at lower energies. For $E>20 \mathrm{MeV}$, the mean free path is well described by SQLT. At $5 R_{\odot}$ the EQLT mean free path is finite at all energies $E>1 \mathrm{MeV}$ and well described by SQLT above $2 \mathrm{MeV}$. At $10 R_{\odot}$, the two theories agree completely at $E>1 \mathrm{MeV}$.

Overall, the mean free path is extremely small compared to the typical values at interplanetary distances because we ignore the spectral evolution that tends to steepen the wave spectrum. According to Tu \& Marsch (1997), however, cascading only turns on at distances $r>10 R_{\odot}$, so up to this point the mean free path should adequately describe the conditions in a corona heated by the cyclotron sweep mechanism. We expect, however, a significant increase on the interplanetary mean free path due to the loss of wave power cascaded to the dissipation process or to non-scattering modes (see below).

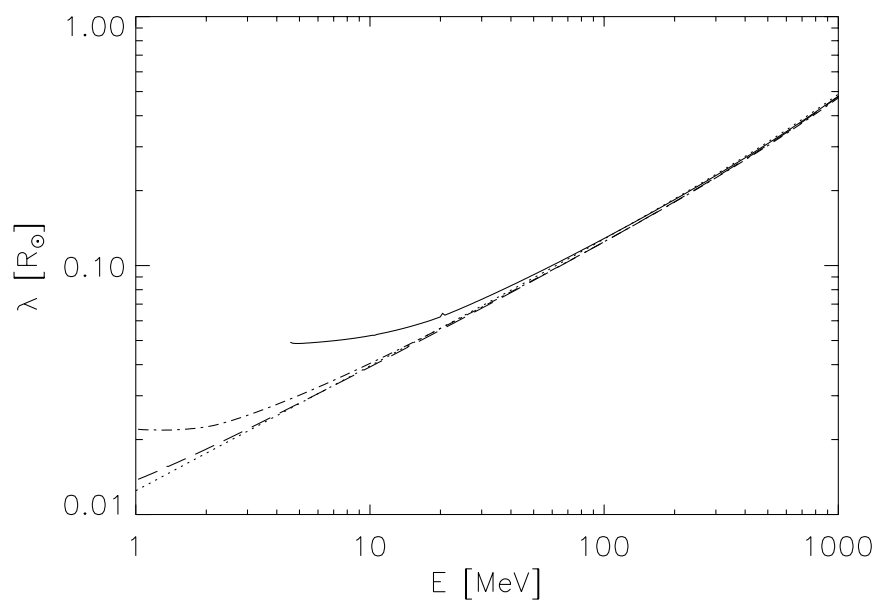

Fig. 4. The EQLT mean free path as a function of energy in solar corona for distances $r=2$ (solid curve), 5 (dash-dotted curve), and $10 R_{\odot}$ (dashed curve). The resonant part of the mean free path at $10 R_{\odot}$ is also shown (dotted curve)

Of course, the presented results are dependent on the choice of some critical parameters in the model. The wave spectrum is the most important parameter controlling the mean free path. We have used an overall level of the fluctuations that corresponds to an extrapolation of the typical $f^{-1}$ spectra observed at low frequencies in the interplanetary medium. Since this, alone, is not enough to produce high-speed winds in the cyclotron sweep mechanism, we regard our choice to be a conservative one.

For the spectral termination frequency $f_{\mathrm{H}}$, we use a law that takes account of the low plasma beta value in the corona $\left(\beta_{\mathrm{p}} \lesssim 0.15\right)$ and agrees well with the observed values of interplanetary dissipation frequencies near $1 \mathrm{~Hz}$. Note also that our choice of $f_{\mathrm{H}}=f_{\mathrm{p}} / 2$ at $r<20 R_{\odot}$ is in accordance with the observed strong heating of $\mathrm{He}^{2+}$ ions, and agrees with the choice of Hollweg (2000). The width of the SQLT resonance gap is inversely proportional to $f_{\mathrm{H}}$; had we taken the same value $(0.1)$ as Tu \& Marsch (1997) for $\alpha_{\mathrm{f}}$ we would have ended up with a $\mu_{\mathrm{c}}$ of about 5 times as large as in the present model. This would be partly compensated if we also included the spectral humps of Tu \& Marsch (1997), which they introduce at high frequencies to drive the fast wind, but we may estimate the energy axis to shift by a factor of $\sim 20$ towards higher energies for the dissipation frequency of $\mathrm{Tu} \&$ Marsch. Another parameter affecting the calculation in a similar way is the Alfvén speed. We have used a radial magnetic field model giving a rather weak field at the coronal base $(1.3 \mathrm{G})$. For a fixed interplanetary field value, super-radial flux-tube expansion enlarges the coronal base field, and if this is not compensated with a density increase, we end up with a large Alfvén speed and $\mu_{\mathrm{c}}$. However, more realistic modeling of the spectrum in the dissipation range including local replenishment of the waves by unstable particle distributions (e.g., streaming, hot minor plasma ions, Li \& Habbal 1999, or the energetic particles themselves, $\mathrm{Ng}$ \& Reames 1994) or by cascading, or even a slight amount of wave reflection would increase scattering in the gap to a 
level that would provide a necessary amount of scattering for the energetic particles to cross $\mu=0$.

Let us estimate the implications of this wave-heating mechanism on particle acceleration in solar corona. First, we note that a shell of extremely small mean free path is located close to the solar surface, favorable for acceleration by coronal shock waves observed as metric type II bursts in many gradual solar energetic particle events. Chromospheric Moreton waves have also been associated with proton acceleration/release at a wide range of solar longitudes (Cliver et al. 1995), and recent observations of coronal Moreton waves (e.g., Torsti et al. 1999) show how large perturbations related to particle acceleration travel far away from the active regions. If we can regard these wave fronts as shocks, the short mean free path gives them a chance to efficiently accelerate particles on open field lines. Alternatively, these coherent, large-amplitude wave fronts may interact with the pre-existing high-frequency Alfvén waves and reflect and amplify them to produce turbulent conditions favorable for stochastic acceleration. In any case, a short mean free path ensures rapid acceleration extending to the $\mathrm{MeV}$ energies and beyond away from active regions but still close to the solar surface.

Further, the short mean free paths between 5 and 10 solar radii help the acceleration of energetic ions in CMEdriven shock waves: the characteristic speed attainable by this mechanism, after which we expect spectral softening due to adiabatic effects, is obtained from $\kappa\left(v_{\mathrm{c}}, r\right) / u \sim r / 2$ (Vainio et al. 2000) yielding $v_{\mathrm{c}}=u 3 r / 2 \lambda\left(r, v_{\mathrm{c}}\right)$. Using $\lambda=0.3(v / c) R_{\odot}$ (see Fig. 3) as a typical coronal mean free path gives $v_{\mathrm{c}}^{2}=5 u \mathrm{cr} / R_{\odot}$, which for $u \sim 10^{-3} \mathrm{c}$ yields a characteristic energy at $r=10 R_{\odot}$ of $E_{\mathrm{c}}=\frac{1}{2} m_{\mathrm{p}} v_{\mathrm{c}}^{2} \sim$ $20 \mathrm{MeV}$. Note, however, that in polar coronal holes with the largest wind speeds, the small densities yield very high Alfvén speeds, far over $1000 \mathrm{~km} \mathrm{~s}^{-1}$ reached already for our flow speed of $550 \mathrm{~km} \mathrm{~s}^{-1}$ at 0.3 AU. Only very few CMEs drive strong shocks in media with such high Alfvén speeds. Thus, the production of energetic particles would still be favored on field lines with smaller and gradually increasing wind speed and larger density, i.e., close to the ecliptic.

Wave-heating models clearly help the acceleration of particles in solar corona. It is also interesting to try to obtain constraints on coronal heating from energetic particles. Can observations of solar energetic particle events tell us, whether the coronal mean free path is as small as the cyclotron sweep mechanism seems to require? Unfortunately, reported measurements of solar energetic particle events at high latitudes are still extremely few, one reason probably being the above mentioned large Alfvén speed in polar corona setting a high threshold speed for the CMEs to drive strong shock waves. Bothmer et al. (1995) report three transient events observed on board Ulysses, while the spacecraft was immersed in the high-speed region over the south pole. These events show very long delay times (several days) for particle fluxes with respect to the launch of the CME from the Sun. However, since the spacecraft was far from the Sun during the events (near
$3.5 \mathrm{AU}$ ), definite conclusions about transport parameters close to the Sun are impossible to make. Thus, we can only state that energetic-particle observations in polar coronal holes do not rule out the cyclotron sweep mechanism as the agent of coronal heating and high-speed solar wind acceleration.

We can also look at observations made in the ecliptic. In particular, are they consistent with the cyclotron sweep as a heating mechanism in solar wind streams of slower speeds, $400-550 \mathrm{~km} \mathrm{~s}^{-1}$, frequently observed near the ecliptic? The fluctuation spectra in near equatorial streams are consistent with a picture where the spectral break point from a $f^{-1}$ to a $f^{-5 / 3}$ behavior occurs at a frequency that is small $\left(\$ 10^{-4} \mathrm{~Hz}\right)$ in low-speed streams and large $\left(\sim 10^{-3} \mathrm{~Hz}\right)$ in high speed streams but showing similar power levels at frequencies below the spectral break point (Marsch 1991b). Thus, the fluctuation spectra seem consistent with a cascade operating - faster in the slow wind - on a similar amount of initial wave power in all types of streams. In the interplanetary medium, one observes a nearly adiabatic expansion of the gas from $3 R_{\odot}$ to $1 \mathrm{AU}$ in streams with speeds less than $300 \mathrm{~km} \mathrm{~s}^{-1}$ (Marsch 1991a). Already at speeds $300-400 \mathrm{~km} \mathrm{~s}^{-1}$ the gas seems to be heated considerably on its way from the Sun to $0.3 \mathrm{AU}$ and beyond. A clear trend of increasing heating with increasing wind speed is obtained, and dissipation of Alfvénic fluctuations seems to provide a consistent source for this heating (Marsch 1991a). As a parametric study by Marsch \& Tu (1997) indicates (see also Sandbaek \& Leer 1994), the intermediate speeds could be obtained by the sweeping mechanism if the coronal base density is high and if the wave spectrum does not posses the bulges needed for the fastest wind speeds (and already neglected in our calculation).

Could energetic-particle observations then tell us, how far the waves propagate until the cascading switches on? The mean free paths of the order of $\lambda \lesssim 0.15 R_{\odot}$ at $r \leq$ $10 R_{\odot}$ and $E<100 \mathrm{MeV}$ (see Fig. 3) imply time scales of the order of $\tau \approx r^{2} / 2 v \lambda \gtrsim\left(9 R_{\odot}\right)^{2} /\left(0.3 R_{\odot} v\right) \approx 300 R_{\odot} / v$ for protons to diffusively leak out from the corona, if they are released near the solar surface. Since convection at Alfvén speed speeds up the transport for low-energy protons, this time scale should be representative also at low energies - although the mean free path increases with energy. If cascading rapidly enlarges the mean free path in interplanetary space, this time lag is nicely in the limit of observations (see, e.g., Torsti et al. 1998).

In fact, impulsive and gradual events show differences that are consistent with some Alfvénic heating and solar-wind acceleration on ecliptic open field lines: the typical speed of the solar wind emitted from the flare site and observed at $1 \mathrm{AU}$ in ${ }^{3} \mathrm{He}$-rich particle events is $390 \pm 15 \mathrm{~km} \mathrm{~s}^{-1}$ and shows a clear minimum above the flare site; for events with normal abundances it is $450 \pm 30 \mathrm{~km} \mathrm{~s}^{-1}$ with no apparent structure around the flare site found (Slivka et al. 1984; Kocharov \& Kocharov 1984). In addition, gradual events can be well fitted with much smaller interplanetary mean free paths than 
impulsive events ( $\sim 0.1$ AU vs. $\sim 1$ AU, respectively) as noted by Kahler (1994). Note also, that our result of the second mean free path minimum is consistent with the observations of gradual events having injections of $>10 \mathrm{MeV}$ protons to the interplanetary medium at times when the related CME is at $5-15 R_{\odot}$ above the solar surface (Kahler $1993,1994)$, although these observations include also the largest gradual events with significant generation of waves by the particles themselves. Nevertheless, from energeticparticle point of view, the cyclotron sweep mechanism can be responsible for the low-coronal heating on open ecliptic field lines, provided that the cascading efficiently removes wave power from the scattering waves by the time they arrive at distances $r \gtrsim 10 R_{\odot}$.

For very large solar energetic-particle events, selfgenerated waves provide a consistent model of the interplanetary transport of particles (Reames et al. 2000) and their acceleration by strong shock waves (Zank et al. 2000). Vainio et al. (2000) concluded that the mean free path of relativistic protons upstream the shock can be at most $\lambda \sim 10^{-3} r$ if they are to be produced efficiently by diffusive shock acceleration. Their study also showed that simultaneously observed interplanetary and interacting (as deduced from secondary emissions) protons can be modeled by arranging the turbulence spatially around the shock in a stationary manner indicating the importance of self-consistent modeling. However, in small events without relativistic protons our test-particle model becomes attractive.

Good examples of small gradual events were presented by Torsti et al. (1998) who analyzed energetic (1-50 MeV) proton intensities at 1 AU related to four Earth-directed CMEs during the first half of 1997 using SOHO observations. Energetic protons measured by ERNE experiment were shown to be correlated with the presence of a coronal Moreton wave: two events with such a wave observed showed orders of magnitude larger proton fluxes at $>10 \mathrm{MeV}$ energies relative to the two events lacking this correlation. The same correlations regarded the existence of X-ray flares and metric type II bursts in the events. This implies, as stressed by the authors, the importance of particle acceleration below $2 R_{\odot}$. Further acceleration and/or trapping of particles may still, of course, occur further away from the Sun. A more detailed analysis of these events by Anttila \& Sahla (2000) revealed that protons were injected from the CME-driven shocks up to $r \gtrsim 10 R_{\odot}$ during these events with spectra that had exponential roll over at energies above $15 \mathrm{MeV}$, in agreement with our calculated mean free path.

Although it is not directly related to the mean free path of energetic particles, one point in our calculation deserves a comment: the dissipation frequency, as calculated from Eq. (4), has a local minimum very close to the solar surface at $\sim 1.3 \mathrm{kHz}$. This means that until the calculated $f_{\mathrm{H}}$ reaches this value again at $r \approx 1.7 R_{\odot}$, no heating by the cyclotron sweep mechanism can occur. This happens, because the plasma beta decreases rapidly as a function of distance and the particles in the low-beta region are too cold to damp the waves propagated there. Thus, in light of our study, a more involved damping frequency taking also account of the plasma beta should be used in the cyclotron sweep model. Also the dispersive effects and the effects of oblique wave vectors (leading to efficient damping by Čerenkov resonances) in case of non-radial fields need to be carefully studied, although the existence of oblique waves in amounts needed by Tu \& Marsch (1997) is questioned by Hollweg (2000), because the density fluctuations they imply seem too large when compared to the observations. Also the absorption of waves by minor ions (Cranmer 2000) seems to be too large for cyclotron sweep to heat protons, although fully kinetic (non-Maxwellian) modeling still waits to be performed on this subject. We refer the reader to Isenberg et al. (2000) for distribution functions resulting from such an analysis, and point out that interesting results can be obtained also from testparticle calculations (e.g., Hollweg 1999). Proceeding with such studies should reveal, if the cyclotron sweep mechanism needs cascading to help to heat the low corona. Or alternatively, as proposed by Leamon et al. (2000), is (most of) the wave power first cascaded to perpendicular wavenumbers to develop a $2 \mathrm{D}$-turbulence component, and dissipated in current sheets resulting from this cascade?

Since the 2D fluctuations scatter the energetic particles much less-effectively than the parallel propagating Alfvén waves (Bieber et al. 1994), our results of the mean free path imply that cascading of Alfvén waves into this mode might take place at the edges of open flux tubes adjacent to the closed-field regions to produce strong initial heating, but leaving the solar wind without interplanetary heating and the acceleration it gets from the from the Alfvén-wave pressure gradient. The remnants of the 2D turbulence would constitute the major part of the observed turbulence in the interplanetary medium. As a result, a slow wind would develop, with an enlarged mean free path of energetic particles, consistent with the results of Bieber at al. (1994). Further inside the open-field region, the perpendicular cascade would operate somewhat slower because of the smaller gradients in the perpendicular direction. This would leave enough Alfvén waves beyond the sonic point to continuously accelerate the solar wind to a larger speed, to provide interplanetary heating, and also for energetic particles to be efficiently (re-)accelerated by coronal shocks. Due to the large coronal base density in these regions (resulting, perhaps, from the strong heating in low corona leading to a large heat flux from the corona to the transition region), the final wind speed would be typically $400-500 \mathrm{~km} \mathrm{~s}^{-1}$ in these flow tubes. Deep in (the inter-plume regions of) the coronal holes, the perpendicular cascade would not be able to destroy the Alfvén waves. This would result in a fast wind and high Alfvén speed - the latter would explain the apparent lack of prominent energetic-particle emission from these sites, although perpendicular diffusion in the turbulent sheath region between the shock and the driver CME should be capable of spreading the particles far from the 
sites of their original acceleration to be detected later also at high latitudes.

\section{Conclusion}

We have calculated the EQLT mean free path of energetic protons in a corona heated by the cyclotron sweep mechanism for a $f^{-1}$ spectrum of WKB Alfvén waves extended up to a dissipation frequency below $1 \mathrm{kHz}$ with an initial frequency-integrated velocity amplitude of $20 \mathrm{~km} \mathrm{~s}^{-1}$. It is shown, that the EQLT mean free path has a nonmonotonous behavior as a function of distance and energy showing a maximum near $r \sim 2 R_{\odot}$, where it attains infinite values for particles of low speed $(E \lesssim 4 \mathrm{MeV} / \mathrm{n})$. Two local minima of $\lambda$ are obtained (i) at the solar surface and (ii) around $10 R_{\odot}$ provided that the waves survive there without being cascaded to the dissipation process or to other (less scattering) modes. In case cascading and/or damping operates rapidly in low corona, a much larger mean free path, perhaps without the second minimum of $\lambda$, would result. This scenario would be consistent with the almost scatter-free conditions and slow solar wind speeds observed above the sites of ${ }^{3} \mathrm{He}$-rich flares, and with more diffusive transport in higher-speed winds prevailing in case of gradual events.

Comparing the calculation to observations of small gradual particle events by Torsti et al. (1998) and Anttila \& Sahla (2000), we find that a preferred region of acceleration is the shell of small mean free path near the solar surface. Further diffusive acceleration by a CME-driven shock on open coronal field lines is shown to be capable of producing/maintaining power law proton spectra extending up to tens of MeVs at altitudes $r \lesssim 10 R_{\odot}$, even without waves of self-generated origin. Since they (probably) have lower values of the Alfvén speed, intermediate-speed (400-500 $\left.\mathrm{km} \mathrm{s}^{-1}\right)$ streams would be more favorable sites for shock acceleration than the fastest streams.

Our study shows that energetic particle observations in interplanetary space are consistent with the picture of polar coronal holes being heated by the cyclotron sweep mechanism. Winds emitted from open-field regions near the ecliptic may also be heated by high-frequency Alfvén waves provided either (i) that the initial wave power on these field lines is much smaller than in polar regions or (ii) that perpendicular cascading and/or damping operates fast enough to remove the power from Alfvén waves by the time the plasma is near $10 R_{\odot}$. The former possibility may exclude the acceleration of the energetic particles by diffusive shock acceleration in the test-particle approximation because of the lack of scattering in the shock's upstream region. It is also not consistent with recent groundbased and SOHO observations (Raju et al. 2000) showing similar non-thermal velocities in coronal holes and surrounding quiet regions in low corona (below $1.03 R_{\odot}$ ). A coronal-heating scenario is discussed, where a perpendicular cascade operates at the edges of open-field flux tubes to remove nearly all power from the Alfvén waves. This would result in a slow solar wind and a large mean free path for energetic particles; however, strong non-linear interactions and (quasi-) 2D fluctuations would help to close up the resonance gap more efficiently than the EQLT, so the mean free path at the lowest energies would decrease from the present prediction (see, e.g., Bieber et al. 1994). Somewhat away from the edges, a fair fraction of the waves would survive the perpendicular cascade, accelerate the wind to intermediate speeds, and give enough scattering for the particles to be (re-)accelerated by coronal shocks to high energies.

Finally, we point out that energetic-particle measurements can clearly give their contribution to the studies of coronal heating with the upcoming Solar Orbiter mission, where high-resolution data can be expected from coronal holes at small distances (0.2 AU) from the Sun at latitudes up to 38 degrees.

Acknowledgements. The authors wish to acknowledge helpful discussions with L. Kocharov.

\section{Appendix A: Pitch-angle scattering in EQLT}

We apply EQLT in a simplified case, where the turbulence consists of Alfvén waves propagating in one direction. In the frame of the waves, thus, dispersion relation $\omega=0$ applies, and the pitch-angle diffusion coefficient is given by (Ng \& Reames 1995)

$$
\begin{aligned}
D_{\mu \mu} & =\frac{1}{4} \frac{\Omega^{2}}{B^{2}}\left(1-\mu^{2}\right) \sum_{n=0}^{2} \int_{-\infty}^{+\infty} \mathrm{d} k I_{n}(k) R_{\mu \mu}^{n}(k, v, \mu) \\
& \equiv \frac{\Omega}{2}\left(1-\mu^{2}\right) \sum_{n=0}^{2} \Phi_{n}(\mu, v),
\end{aligned}
$$

where $I_{n}(k)$ is the spectrum of waves responsible for the scattering by process $n$, and, keeping only the lowest order terms in small parameters $b_{\perp}$ and $\mu_{\mathrm{c}}$,

$$
\begin{aligned}
& R_{\mu \mu}^{0}=J_{1}^{2}(\rho) \Delta_{0}(k, v, \mu) \\
& R_{\mu \mu}^{1}=J_{0}^{2}(\rho) \Delta_{1}(k, v, \mu) \\
& R_{\mu \mu}^{2}=J_{1}^{2}(\rho) \Delta_{2}(k, v, \mu)
\end{aligned}
$$

are the resonance functions giving the strength of the wave-particle interactions. Above, $\rho=b_{\perp} \sqrt{1-\mu^{2}} k r_{\mathrm{L}}$, and

$$
\begin{aligned}
\Delta_{n}(k, v, \mu) & =\frac{2 H\left\{D_{n}(k, v, \mu)\right\}}{\sqrt{D_{n}(k, v, \mu)}} \\
D_{n}(k, v, \mu) & =v^{2}\left(b_{\perp}^{2}-\mu^{2}\right)\left(k-k_{n+}\right)\left(k-k_{n-}\right) \\
k_{n \pm}(v, \mu) & =-\frac{n}{r_{\mathrm{L}}} \frac{1}{\mu \mp b_{\perp} \sqrt{1-\mu^{2}}}
\end{aligned}
$$

In their original formulation, $\mathrm{Ng} \&$ Reames took $I_{n}(k)=$ $I(k)$ for all $n$. This is completely valid for the gyroresonant interactions, $n=1,2$. We note, however, that for the $n=0$ resonance, the assumption of uncorrelated phases of the waves is crucial since this resonance only appears if the field fluctuates in the direction of local $\boldsymbol{B}$. For a purely circularly-polarized perturbation 
$\delta \boldsymbol{B}, \delta \boldsymbol{B} \cdot \boldsymbol{B} / B=\delta B^{2} / \sqrt{B_{0}^{2}+\delta B^{2}}$ is constant and no $n=0$ resonances appear. If the wave-field starting from the corona is purely Alfvénic, the phases of the Fourier components have to get mixed during the propagation to produce magnitude fluctuations. This, however, occurs already in the linear model, since left- and right-handed waves have slightly different phase speeds given by

$$
\frac{2 \pi f}{|k|}=\left(V+V_{\mathrm{A}}\right) \pm V_{\mathrm{A}} \frac{f}{2 f_{\mathrm{p}}}
$$

valid for $f<f_{\mathrm{p}} / 2$ (or $|k|<\Omega_{\mathrm{p}} / 2 V_{\mathrm{A}}$ ). At constant frequency, left- and right-handed waves start to develop large phase shifts, when

$2 \pi f \int_{R_{\odot}}^{r} \frac{\mathrm{d} r^{\prime}}{V\left(r^{\prime}\right)+V_{\mathrm{A}}\left(r^{\prime}\right)} \frac{2 \pi f}{\left[M\left(r^{\prime}\right)+1\right]^{2} 2 \Omega_{\mathrm{p}}\left(r^{\prime}\right)} \sim 1$.

We estimate the wavenumber $k_{\mathrm{c}}$, above which the waves have random phases, as

$k_{\mathrm{c}}^{-2}=\left(V+V_{\mathrm{A}}\right)^{2} \int_{R_{\odot}}^{r} \frac{\mathrm{d} r^{\prime}}{2 V_{\mathrm{A}}\left(r^{\prime}\right)\left[M\left(r^{\prime}\right)+1\right]^{3} \Omega_{\mathrm{p}}\left(r^{\prime}\right)}$.

We, thus, take $I_{0}(k)=H\left(|k|-k_{\mathrm{c}}\right) I(k)$. As can be seen from Fig. 2, the phase randomization begins at frequencies $k_{\mathrm{c}} /\left[2 \pi\left(V+V_{\mathrm{A}}\right)\right]$ that are from two to three orders of magnitude below the cutoff frequency in our model, so locally, the dispersion relation is still very close to Alfvénic even for most of these waves.

The resonance condition $\mu=-1 /\left(k r_{\mathrm{L}}\right)$ in the SQLT is replaced by the conditions $\left|\mu+n /\left(k r_{\mathrm{L}}\right)\right|<$ $b_{\perp} \sqrt{1-\left(n / k r_{\mathrm{L}}\right)^{2}},|k| \geq n / r_{\mathrm{L}}$ in EQLT. If $\mu_{\mathrm{c}}<b_{\perp}$, $n=1$ resonance broadening yields finite scattering over the whole velocity space. But taking the $n=0$ resonance into account shows that the EQLT is capable of closing the resonance gap for particles for which the broadening of the $n=1$ resonance yields scattering inside the $n=0$ resonance range, i.e., if $\mu_{\mathrm{c}}<2 b_{\perp}$ to the lowest order in $b_{\perp}$. For other particles, even the EQLT produces infinite mean free paths.

The $n=0$ resonance function, for $|\mu|<b_{\perp}$ and $I_{0}(k)=$ $\frac{1}{2} R(r) /|k|$ in $k_{\mathrm{c}}<|k|<k_{\mathrm{H}}=1 / \mu_{\mathrm{c}} r_{\mathrm{L}}$ with $k_{\mathrm{H}} \gg k_{\mathrm{c}}$, gives

$\Phi_{0}=\frac{R}{B^{2}} \frac{b_{\perp}}{\sqrt{b_{\perp}^{2}-\mu^{2}}}\left[J i_{1}\left(b_{\perp} / \mu_{\mathrm{c}}\right)-J i_{1}\left\{x_{\mathrm{c}} b_{\perp} / \mu_{\mathrm{c}}\right\}\right]$,

where $x_{\mathrm{c}}=k_{\mathrm{c}} / k_{\mathrm{H}}$ and the function $J i_{1}(z)$ is defined as

$J i_{1}(z)=\int_{0}^{z} \frac{J_{1}^{2}(y)}{y^{2}} \mathrm{~d} y$

The maximum value for $\Phi_{0}$ is acquired for $x_{\mathrm{c}} \ll$ $\mu_{\mathrm{c}} / b_{\perp} \ll 1$, when the brackets give a value of $J i_{1}(\infty) \approx$ 0.42 .

Outside $|\mu|<b_{\perp}$, another contribution to $\lambda$ comes from the range of modified gyro-resonances, $n \mu_{\mathrm{c}}-b_{\perp}<$ $|\mu|<n \mu_{\mathrm{c}}+b_{\perp}$. There, $1-\mu^{2} \approx 1$ and

$D_{n} \approx \Omega^{2}\left(\mu^{2}-b_{\perp}^{2}\right)\left(x-x_{-}\right)\left(x_{+}-x\right) / \mu_{\mathrm{c}}^{2}$, where $x_{ \pm}=\mu_{\mathrm{c}} /\left(\mu \mp b_{\perp}\right)$ and $x=-k / k_{\mathrm{H}}$. The $n=1$ resonance function is

$R_{\mu \mu}^{1} \approx \frac{2 \mu_{\mathrm{c}} H\left\{\left(\mu^{2}-b_{\perp}^{2}\right)\left(x-x_{-}\right)\left(x_{+}-x\right)\right\}}{\Omega \sqrt{\left(\mu^{2}-b_{\perp}^{2}\right)\left(x-x_{-}\right)\left(x_{+}-x\right)}} J_{0}^{2}\left(x b_{\perp} / \mu_{\mathrm{c}}\right)$.

This gives, for $\mu_{\mathrm{c}}+b_{\perp}>\mu>\mu_{\mathrm{c}}-b_{\perp}>0$ (with $\left.J_{0}^{2}\left(x b_{\perp} / \mu_{\mathrm{c}}\right) \approx 1\right)$

$$
\begin{aligned}
\Phi_{1} & \approx \frac{\mu_{\mathrm{c}} R(r)}{2 B^{2} \sqrt{\left|\mu^{2}-b_{\perp}^{2}\right|}} \int_{x_{-}}^{x_{+}} \mathrm{d} x \frac{H(1-x)}{x \sqrt{\left(x-x_{-}\right)\left|x_{+}-x\right|}} \\
& =\frac{R(r)}{2 B^{2}} \arccos \frac{\mu_{\mathrm{c}}-\mu}{b_{\perp}}
\end{aligned}
$$

where the $\arccos X$ function should be replaced by $0(\pi)$ if $X>1(X<-1)$. Despite of the approximations, this converges towards the SQLT value at $\mu \rightarrow \mu_{\mathrm{c}}+b_{\perp}$. If $\mu_{\mathrm{c}}<b_{\perp}$, the scattering rate becomes modified including contributions from the range $x \in\left(-1, x_{+}\right)$, giving another contribution obtained from Eq. (A.4) by substitution $\mu \rightarrow-\mu$. This result, however, does not hold for $\mu_{\mathrm{c}} / b_{\perp}$ much less than unity, because of the crude Bessel-function approximation. Noting that most of the contribution to the integral is coming from near $x \rightarrow x_{ \pm}$, we take the Bessel function out from the integrand and obtain

$$
\begin{aligned}
\Phi_{1} \approx & \frac{R(r)}{2 B^{2}}\left[J_{0}^{2}\left(\frac{b_{\perp}}{b_{\perp}+\mu}\right) \arccos \frac{\mu_{\mathrm{c}}-\mu}{b_{\perp}}\right. \\
& \left.+J_{0}^{2}\left(\frac{b_{\perp}}{b_{\perp}-\mu}\right) \arccos \frac{\mu_{\mathrm{c}}+\mu}{b_{\perp}}\right] .
\end{aligned}
$$

Noting the similarities between $R_{\mu \mu}^{1}$ and $R_{\mu \mu}^{2}$, we may write

$$
\begin{aligned}
\Phi_{2} \approx & \frac{R(r)}{2 B^{2}}\left[J_{1}^{2}\left(\frac{2 b_{\perp}}{b_{\perp}+\mu}\right) \arccos \frac{2 \mu_{\mathrm{c}}-\mu}{b_{\perp}}\right. \\
& \left.+J_{1}^{2}\left(\frac{2 b_{\perp}}{b_{\perp}-\mu}\right) \arccos \frac{2 \mu_{\mathrm{c}}+\mu}{b_{\perp}}\right] .
\end{aligned}
$$

In Eqs. (A.5) and (A.6), the $\arccos X$ function should again be replaced by $0(\pi)$ if $X>1(X<-1)$.

\section{References}

Anttila, A., \& Sahla, T. 2000, Ann. Geophys., 18, 1373

Bieber, J. W., Matthaeus, W. H., Smith, C. W., et al. 1994 ApJ, 420, 294

Bothmer, V., Marsden, R. G., Sanderson, T. R., et al. 1995, Geophys. Res. Lett., 22, 3369

Cliver, E. W., Kahler, S. W., Neidig, D. F., et al. 1995, Proc. 24th Internat. Cosmic Ray Conf., 4, 257

Cranmer, S. R. 2000, ApJ, 532, 1197

Hollweg, J. V. 1999, J. Geophys. Res., 104, 24781

Hollweg, J. V. 2000, J. Geophys. Res., 105, 7573

Horbury, T. S., Balogh, A., Forsyth, R. J., \& Smith, E. J. 1996, A\&A, 316, 333

Hu, Y. Q., Habbal, S. R., \& Li, X. 1999, J. Geophys. Res., 104, 24819

Isenberg, P. A., Lee, M. A., \& Hollweg, J. V. 2000, Solar Phys., 193, 247

Kahler, S. W. 1993, J. Geophys. Res., 98, 5607 
Kahler, S. W. 1994, ApJ, 428, 837

Kobak, T., \& Ostrowski, M. 2000, MNRAS, 317, 973

Kocharov, L. G., \& Kocharov, G. E. 1984, SSRv, 38, 89

Kocharov, L., Kovaltsov, G. A., Laitinen, T., Mäkelä, P., \& Torsti, J. 1999, ApJ, 521, 898

Leamon, R. J., Matthaeus, W. H., Smith, C. W., et al. 2000, ApJ, 537, 192L

Li, X., \& Habbal, S. R. 1999, Solar Phys., 190, 485

Marsch, E. 1991a, in Physics of the Inner Heliosphere II, ed. R. Schwenn, \& E. Marsch (Springer-Verlag, Berlin Heidelberg), 45

Marsch, E. 1991b, in Physics of the Inner Heliosphere II, ed. R. Schwenn, \& E. Marsch (Springer-Verlag, Berlin Heidelberg), 159

Marsch, E., \& Tu, C.-Y. 1997, A\&A, 319, L17

McKenzie, J. F., Banaszkiewicz, M., \& Axford, W. I. 1995, A\&A, 303, L45

Ng, C. K., \& Reames, D. V. 1994, ApJ, 424, 1032

Ng, C. K., \& Reames, D. V. 1995, ApJ, 453, 890
Raju, K. P., Sakurai, T., Ichimoto, K., \& Singh, J. 2000, ApJ, 543, 1044

Reames, D. V., Ng, C. K., \& Tylka, A. J. 2000, ApJ, 531, L83

Sandbaek, Oe., \& Leer, E. 1994, ApJ, 423, 500

Schlickeiser, R. 1989, ApJ, 336, 243

Schlickeiser, R., \& Achatz, U. 1993, J. Plasma Phys., 49, 63

Slivka, M., Kocharov, L. G., \& Dvoryanchikov, Y. V. 1984, Acta Phys. Slov., 34, 363

Stix, T. H. 1962, The Theory of Plasma Waves (McGraw-Hill, New York), 195

Torsti, J., Anttila, A., Kocharov, L., et al. 1998, Geophys. Res. Lett., 25, 2525

Torsti, J., Kocharov, L. G., Teittinen, M., \& Thompson, B. J. 1999, ApJ, 510, 460

Tu, C.-Y., \& Marsch, E. 1997, Solar Phys., 171, 363

Vainio, R. 2000, ApJS, 131, 519

Vainio, R., Kocharov, L., \& Laitinen, T. 2000, ApJ, 528, 1015

Zank, G. P., Rice, W. K. M., \& Wu, C. C. 2000, J. Geophys. Res., 105, 25079 